\title{
Achados of talmológicos em 28 crianças portadoras da seqüência de Möebius
}

\author{
Möebius sequence: clinical ophthalmological findings in 28 cases
}

\author{
Laura Patrícia Ferreira Santos ${ }^{1}$ \\ Liana Maria V. de Oliveira Ventura ${ }^{2}$ \\ Henderson Celestino de Almeida ${ }^{3}$ \\ Marilyn Miller ${ }^{4}$ \\ Ana Carolina Colier 5
}

${ }^{1}$ Residente em Oftalmologia na Fundação Altino Ventura. 2 Doutora em Oftalmologia Pediátrica e Estrabismo pela Universidade Federal de Minas Gerais (UFMG).

${ }^{3}$ Coordenador do Departamento de Estrabismo da Universidade Federal de Minas Gerais (UFMG).

${ }^{4}$ Coordenadora do Departamento de Oftalmologia da Universidade de Illinois, Chicago, EUA.

${ }^{5}$ Oftalmologista Pediátrica do Instituto de Olhos do Recife.

Endereço para correspondência: FAV - Fundação Altino Ventura, Rua da Soledade, 170 - Recife (PE) CEP 50070-040

E-mail: fav@fundacaoaltinoventura.org.br Recebido para publicação em 31.03.2003 Versão revisada recebida em 10.11.2003 Aprovação em 20.01.2004

Nota Editorial: Pela análise deste trabalho e por sua anuência na divulgação desta nota, agradecemos à Dra. Ana Tereza Ramos Moreira.

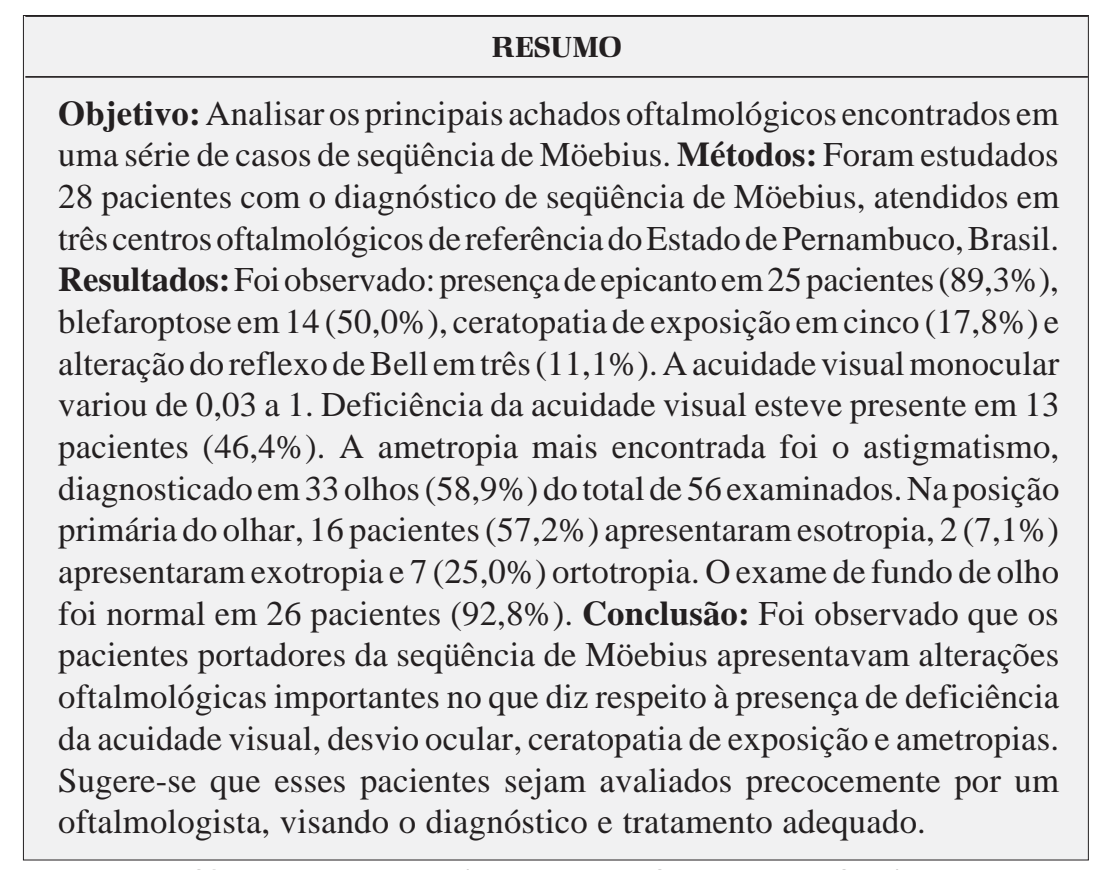

Descritores: Síndrome de Möebius/complicações; Oftalmopatias; Olho/patologia, Acuidade visual; Criança

\section{INTRODUĈ̃̃O}

A seqüência de Möebius, definida como uma paresia ou paralisia congênita do sexto e sétimo nervos cranianos, está freqüentemente associada à paralisia de outros nervos cranianos, anomalias craniofaciais e músculoesqueléticas $^{(1-5)}$. Sua etiopatogenia ainda não está perfeitamente esclarecida. A teoria mais aceita sugere que existiria um mecanismo de insuficiência vascular em idade gestacional precoce, desencadeada por uma série de fatores heterogêneos, dentre os quais se destacam anomalias cromossômicas $^{(6-8)}$, exposição a agentes ambientais como a talidomida ${ }^{(9)}$, cocaína ${ }^{(10)}$, benzodiazepnicos $^{(11)}$, álcool ${ }^{(3)}$ e, em especial, o misoprostol ${ }^{(12-16)}$, o que levaria a produção da seqüência de Möebius ${ }^{(5,17-18)}$.

Em virtude das alterações multisistêmicas da seqüência de Möebius, observa-se que a conduta apropriada varia de acordo com a gravidade e tipo das malformações presentes. A conduta oftalmológica deve ser dirigida para o estrabismo e para exposição corneana, essa última podendo ocorrer, principalmente ao dormir, devido ao acometimento do VII nervo craniano, devendo-se também tratar as ametropias e a ambliopia, se presente ${ }^{(3)}$.

Freqüentemente os genitores e pacientes com a seqüência de Möebius 
buscam o diagnóstico e tratamento através de profissionais que, devido a pouca freqüência da doença, têm dificuldade em diagnosticar e conduzir o caso. O oftalmologista muitas vezes pode ser o primeiro contato dessas crianças com a área médica. Assim sendo, os autores tiveram por objetivo analisar os principais achados oftalmológicos encontrados em uma série de 28 pacientes com a seqüência de Möebius, colaborando para melhor conhecimento desta doença, facilitando o diagnóstico e conduta precoce adequada.

\section{MÉTODOS}

Foi realizado um estudo em uma série de 28 pacientes com o diagnóstico de seqüência de Möebius, atendidos em três centros oftalmológicos de referência do Estado de Pernambuco: Fundação Altino Ventura, Hospital de Olhos de Pernambuco e Associação de Auxílio à Criança Deficiente (AACD) ${ }^{(19)}$.

Os pacientes foram incluídos quando apresentavam paresia ou paralisia congênita dos sexto e sétimo nervos cranianos, acometidos de forma uni ou bilateral ${ }^{(3)}$. As anormalidades de tronco, membros e crânio-faciais, embora freqüentes, não foram considerados como critérios de inclusão.

A idade dos pacientes ao exame variou de um a 141 meses (Média=60,5 meses, Erro padrão da média= 7,7). Doze pacientes (42,9\%) eram do gênero masculino e $16(57,1 \%)$ do feminino. Foram avaliados dados gestacionais, antecedentes pessoais, história familiar e dados do exame oftalmológico.

A acuidade visual para longe (AVL), com correção óptica, foi aferida de acordo com a idade do paciente, cooperação e habilidade mental.

A classificação dos erros refrativos foi feita segundo os critérios adotados por Alves ${ }^{(20)}$.

A deficiência da acuidade visual foi definida, nos pacientes cuja AVL foi aferida utilizando-se a tabela de Snellen, como a presença de diferença de AVL nos dois olhos igual ou superior a duas unidades na tabela, ou em casos de acuidade visual inferior a 0,5 , bilateralmente. Nos pacientes com AVL aferida com o método de Teller, foi considerada deficiência da acuidade visual caso a AVL obtida tenha sido inferior ao padrão da normalidade para a faixa etária.

Foi definido a blefaroptose de acordo com os critérios adotados por Soares ${ }^{(21)}$. A mensuração da abertura palpebral foi realizada por dois pesquisadores em momentos distintos.

Foram avaliadas a presença de nistagmo, posição viciosa de cabeça, ducções e versões com especial atenção para limitações dos movimentos oculares e mudanças na abertura palpebral durante os movimentos oculares horizontais. No exame da musculatura extrínseca ocular, a hipofunção muscular foi convencionada variando de $-1 \mathrm{a}-8^{(4)}$. A convergência ocular foi avaliada através do ponto próximo de convergência (PPC).

Foi empregado o teste de Krimsky para a medida do desvio ocular, com o paciente fixando de perto $(33 \mathrm{~cm})$, em posição primária do olhar, infra e supraversão. A anisotropia em “A” foi considerada, como a presença de diferença entre as medidas obtidas em supra e infraversões igual ou maior que 10 dioptrias prismáticas (DP) e em "V" quando maior ou igual a 15 DP.

A biomicroscopia foi realizada em todos os pacientes que cooperaram. Foi pesquisada sensibilidade corneana, lacrimejamento anormal, reflexos pupilares e reflexo de Bell. Foi feita oftalmoscopia indireta e a retinoscopia sob cicloplegia.

As variáveis qualitativas foram expressas por suas freqüências. Possíveis diferenças entre freqüências foram avaliadas pelo teste do qui-quadrado. As variáveis quantitativas foram expressas por suas médias, desvios padrão e erros padrão da média. Foi utilizado o teste $t$ de Student para avaliar possíveis diferenças entre médias. Foi utilizado $p<0,005$ para rejeição da hipótese de nulidade.

\section{RESULTADOS}

Os achados do exame ocular estão expressos na tabela 1.

A acuidade visual monocular variou de 0,03 a 1 . Em dois pacientes $(7,1 \%)$, devido a pouca cooperação, foi aferida a acuidade visual utilizando o padrão de fixação ocular, sendo classificada como Boa, Mantida e Centrada (BMC). Três crianças não colaboraram para a aferição da acuidade visual monocularmente, sendo esta, aferida binocularmente. A deficiência da acuidade visual esteve presente em 13 pacientes (46,4\%). A idade, acuidade visual para longe e a retinoscopia de cada paciente está expressa na tabela 2.

A ametropia mais encontrada foi o astigmatismo, diagnosticado em 33 olhos (58,9\%), do total de 56 examinados, seguido da hipermetropia presente em 19 (33,9\%) e miopia presente em 3 (5,3\%) (Gráfico 1).

No exame estrabológico da musculatura extrínseca ocular na posição primária do olhar, havia esotropia em 16 casos (57,1\%), exotropia em 2 (7,1\%), ortotropia em 7 (25,0\%), desvio

Tabela 1. Achados oftalmológicos em pacientes com seqüencia de
Möebius
$\begin{array}{lcc}\text { Achado ocular } & \text { N } & (\%) \\ \text { Epicanto } & 25 & 89,2 \\ \text { Blefarofimose } & 3 & 10,8 \\ \text { Ptose } & 14 & 50,0 \\ \text { Alterações do reflexo de Bell* } & 3 & 11,1 \\ \text { Ceratopatia de exposição } & 5 & 17,8 \\ \text { Opacidade corneana } & 2 & 7,1 \\ \text { Medida da abertura palpebral* (mm) } & \\ \text { Média } & \text { (OD) } 20 & \text { (OE) } 21 \\ \text { DP } & \text { (OD) } 21 & \text { (OE) } 20 \\ \text { Mín. } & \text { (OD) } 16 & \text { (OE) } 16 \\ \text { Máx. } & \text { (OD) } 25 & \text { (OE) } 24 \\ { }^{* 1} \text { paciente não permitiu o exame } & & \end{array}$




\begin{tabular}{|c|c|c|c|c|c|c|}
\hline \multirow{2}{*}{$\begin{array}{l}\mathrm{N}^{\circ} \\
\text { caso }\end{array}$} & \multirow{2}{*}{$\begin{array}{c}\text { Idade } \\
\text { (meses) }\end{array}$} & \multicolumn{3}{|c|}{ AVL S/C } & \multicolumn{2}{|c|}{ Refração } \\
\hline & & OD & OE & Binocular & OD & OE \\
\hline 1 & 38 & $0,87 \mathrm{~T}$ & $0,61 \mathrm{~T}$ & NA & plano & $+1,00$ \\
\hline 2 & 74 & $0,50 \mathrm{~S}$ & $0,50 \mathrm{~S}$ & NA & $+1,00$ & $+1,00$ \\
\hline 3 & 28 & $0,22 \mathrm{~T}$ & $0,15 \mathrm{~T}$ & NA & $+1,00 \approx-2,00$ a $90^{\circ}$ & $+2,00 \approx-2,00$ a $90^{\circ}$ \\
\hline 4 & 34 & $0,63 \mathrm{~T}$ & $0,32 \mathrm{~T}$ & NA & $+2,00$ & $-1,50 \approx-2,75$ a $90^{\circ}$ \\
\hline 5 & 10 & $0,22 \mathrm{~T}$ & $0,22 \mathrm{~T}$ & NA & $+4,00$ & $+4,00$ \\
\hline 6 & 33 & $0,15 \mathrm{~T}$ & $0,15 \mathrm{~T}$ & NA & $+3,00$ & $+3,00$ \\
\hline 7 & 72 & $1,00 \mathrm{~S}$ & $0,20 \mathrm{~S}$ & NA & $+1,50 \approx-1,00$ a $30^{\circ}$ & $-4,00 \approx-3,50$ a $30^{\circ}$ \\
\hline 8 & 108 & NA & NA & 0,007 TB & $+1,00 \approx-2,00$ a $180^{\circ}$ & $+1,00 \approx-2,00$ a $180^{\circ}$ \\
\hline 9 & 132 & $0,07 \mathrm{~T}$ & $0,03 \mathrm{~T}$ & NA & $+1,00$ & $+1,00$ \\
\hline 10 & 35 & $0,42 \mathrm{~T}$ & $0,42 \mathrm{~T}$ & NA & $+3,00 \approx-1,00$ a $180^{\circ}$ & $+3,00 \approx-0,50$ a $180^{\circ}$ \\
\hline 11 & 115 & $0,80 \mathrm{~S}$ & $0,80 \mathrm{~S}$ & NA & $+4,00 \approx-2,50$ a $180^{\circ}$ & $+3,50 \approx-3,00$ a $180^{\circ}$ \\
\hline 12 & 27 & $0,63 \mathrm{~T}$ & $0,63 \mathrm{~T}$ & NA & Plano $\approx-1,50$ a $150^{\circ}$ & $+2,00 \approx-1,00$ a $30^{\circ}$ \\
\hline 13 & 34 & NA & NA & 0,11 TB & $+0,50 \approx-0,50$ a $150^{\circ}$ & Plano $\approx-0,25$ a $30^{\circ}$ \\
\hline 14 & 16 & $0,11 \mathrm{~T}$ & $0,07 \mathrm{~T}$ & NA & $-0,50 \approx-1,50$ a $180^{\circ}$ & Plano $\approx-0,75$ a $180^{\circ}$ \\
\hline 15 & 32 & NA & NA & 0,22 TB & Plano $\approx-1,00$ a $90^{\circ}$ & Plano $\approx-1,25$ a $90^{\circ}$ \\
\hline 16 & 10 & $0,15 \mathrm{~T}$ & $0,10 \mathrm{~T}$ & NA & $-0,75$ & $-0,75$ \\
\hline 17 & 26 & $0,21 \mathrm{~T}$ & $0,32 \mathrm{~T}$ & NA & $+2,00$ & $+2,00$ \\
\hline 18 & 98 & $1,00 \mathrm{~S}$ & $0,25 \mathrm{~S}$ & NA & $+0,50 \approx-0,50$ a $90^{\circ}$ & $+1,00 \approx-0,50$ a $75^{\circ}$ \\
\hline 19 & 49 & $0,32 \mathrm{~T}$ & $0,15 \mathrm{~T}$ & NA & $-3,00 \approx-2,00$ a $150^{\circ}$ & $-3,75$ \\
\hline 20 & 58 & $0,20 \mathrm{~S}$ & $0,20 \mathrm{~S}$ & NA & $+1,00$ & $+1,00$ \\
\hline 21 & 109 & $1,00 \mathrm{~S}$ & $1,00 \mathrm{~S}$ & NA & $+1,50 \approx-1,00$ a $90^{\circ}$ & $+2,00 \approx-0,50$ a $90^{\circ}$ \\
\hline 22 & 122 & $1,00 \mathrm{~S}$ & $1,00 \mathrm{~S}$ & NA & $+0,50 \approx-2,00$ a $40^{\circ}$ & Plano $\approx-1,25$ a $165^{\circ}$ \\
\hline 23 & 1 & BMC & $\mathrm{BMC}$ & BMC & $+1,50 \approx-1,50$ a $90^{\circ}$ & $+1,00 \approx-1,50$ a $90^{\circ}$ \\
\hline 24 & 95 & $0,80 \mathrm{~S}$ & $0,80 \mathrm{~S}$ & NA & $+1,50 \approx-2,50$ a $155^{\circ}$ & $+0,75 \approx-2,50$ a $50^{\circ}$ \\
\hline 25 & 80 & $1,00 \mathrm{~S}$ & $1,00 \mathrm{~S}$ & NA & $+3,00$ & $+3,00$ \\
\hline 26 & 14 & $0,15 \mathrm{~T}$ & $0,15 \mathrm{~T}$ & NA & $+200 \approx-100$ a $180^{\circ}$ & $+3,00$ \\
\hline 27 & 32 & $\mathrm{BMC}$ & $\mathrm{BMC}$ & BMC & $-6,00 \approx-100$ a $150^{\circ}$ & $-5,00 \approx-100$ a 30 \\
\hline 28 & 48 & $0,11 \mathrm{~T}$ & $0,11 \mathrm{~T}$ & NA & $+2,00$ & $+3,00$ \\
\hline
\end{tabular}

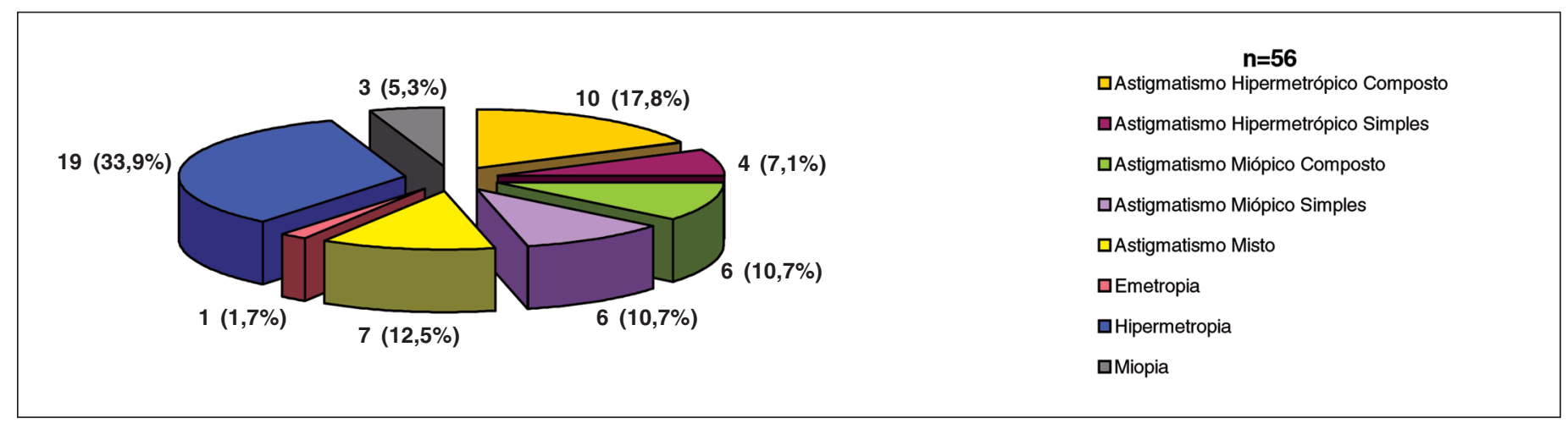

Gráfico 1

vertical simples em $3(10,7 \%)$ e desvio vertical concomitante a esotropia em 4 casos (14,3\%). A anisotropia em " $V$ " foi a mais freqüente, tendo sido encontrada em 9 pacientes (32,1\%).

Foi observada limitação da abdução em todos os casos estudados e convergência ocular próxima do normal (PPC dorso) em 17 casos (60,7\%). Posição viciosa de cabeça esteve presente em 10 casos (35,7\%), fixação cruzada em 10 (35,7\%) e modificação da abertura palpebral em adução em 6 (21,4\%). O grau de rotação da musculatura extraocular está expresso na tabela 3.

O exame de fundo de olho foi normal em 26 pacientes (92,8\%). Em um paciente (3,5\%) foi observado escavação do disco óptico maior que 0,5 bilateralmente e em outro paciente (3,5\%) foi verificado exacerbação da tortuosidade vascular retiniana em ambos os olhos. 


\begin{tabular}{|lccc|}
\hline \multicolumn{4}{|c|}{ Tabela 3. Exame da musculatura extrínseca ocular em pacientes com } \\
seqüência de Möebius \\
MEO & Média & DP & Variação \\
RMD & $-2,6$ & $\pm 1,4$ & $0 /-4$ \\
RLD & $-5,1$ & $\pm 1,4$ & $3 /-8$ \\
RME & $-2,5$ & $\pm 1,5$ & $0 /-4$ \\
RLE & $-5,2$ & $\pm 1,4$ & $3 /-8$ \\
MEO: Musculatura extrínseca ocular; RMD: Reto medial direito; RLD: Reto \\
lateral direito; RME: Reto medial esquerdo; RLE: Reto lateral esquerdo \\
\hline
\end{tabular}

\section{DISCUSSÃO}

As crianças com seqüência de Möebius apresentam importantes alterações oftalmológicas, sendo o estrabismo o achado que mais chama atenção dos genitores. O defeito mais comum dos movimentos oculares está representado pela limitação ou ausência da abdução ${ }^{(22)}$, o que foi encontrado em 100\% da presente análise. Além disso, há relatos de limitação da adução (50,0\%) ou paresia do olhar conjugado horizontal associado ou não a alterações dos movimentos verticais ${ }^{(22)}$, o que está de acordo com os dados obtidos no presente estudo, no qual foi observado restrição da convergência ocular em $29,2 \%$ e desvio vertical em 25,0\% ( $p=0,169)$.

Apesar da paralisia bilateral do VI nervo craniano estar presente na seqüência de Möebius, os pacientes apresentam esotropia em freqüências variáveis indo de menos de 50,0\% dos $\operatorname{casos}^{(23-24)}$ a $68,8 \%{ }^{(25)}, 77,8 \%{ }^{(22)}$ ou $100 \%{ }^{(26)}$ dos pacientes. Na casuística estudada foi observado esodesvio em $57,1 \%$ dos casos em concordância com os dados desses autores ( $p=0,447 / p=0,152 / p=0,07$, respectivamente). A não ocorrência de estrabismo convergente com abdução ausente pode ser atribuída à paresia do músculo reto medial ${ }^{(24)}$. Em relação à presença de exodesvio e ortotropia nesta análise (7,1\% e $25,0 \%$, respectivamente) foi observada semelhança com os dados da literatura que apontam presença de exodesvio em $11,1 \%$ e ortotropia em $11,1 \%{ }^{(22)}(p=0,639 / p=0,448$, respectivamente).

Anisotropia em $\mathrm{V}$ foi mais freqüente estando presente em nove casos (32,1\%), em concordância com os dados que apontam presença deste achado em 50\% da casuística ${ }^{(25)}$ $(p=0,226)$.

Na amostra estudada foi observada fixação cruzada em $37,5 \%$ dos pacientes. Apesar de ter sido admitido que este quadro previne a baixa visual ${ }^{(26)}$, foi encontrada, na presente análise, variação da acuidade visual monocular de 0,03 a 1, com presença de deficiência da acuidade visual em 46,4\% dos pacientes, o que poderia ser atribuído à presença de ametropias, estrabismo, como também a ceratopatia de exposição de repetição. Vale salientar que em três pacientes foi aferida a acuidade visual binocularmente devido à falta de cooperação dos mesmos para aferição monocular. Nestes, foi encontrado distúrbio comportamental autístico em um deles, distúrbio "autismo like" em outro e retardo mental no terceiro paciente ${ }^{(19)}$.
A ocorrência freqüente de blefaroptose na amostra estudada deve refletir a maior acurácia nas mensurações realizadas no presente estudo. Este achado deve representar a real freqüência desta alteração clínica na seqüência de Möebius.

Tem sido observado que existe uma elevada incidência de alterações da função palpebral, presença de lacrimejamento anormal e cicatrizes corneanas em crianças com a seqüência de Möebiuss ${ }^{(22)}$. Há relatos da presença de lagoftalmo simétrico ou assimétrico em freqüências variáveis indo de 100\% da casuística, sendo comum a presença de cicatrizes subepiteliais difusas ${ }^{(22)}$, a $68,8 \%{ }^{(25)}$, o que diverge dos resultados do presente estudo, no qual foi observado lagoftalmia em 14,3\% e ceratopatia de exposição em 17,8\% $(p<0,01)$. Alteração do fenômeno de Bell tem sido descrito em um paciente $(6,3 \%)^{(25)}$, estando associado a leucoma de exposição em ambos os olhos, enquanto que na presente casuística foi observado alteração do fenômeno de Bell em três pacientes $(11,1 \%)$, estando associado a ceratopatia em apenas um desses $(p=1,00)$. Salienta-se a importância deste achado, uma vez que há relato de um caso de ambliopia devido a ceratopatia de exposição bilateral ${ }^{(27)}$.

No presente estudo, foram detectados $89,2 \%$ dos pacientes com prega epicântica bilateral, diferindo dos dados de Henderson que aponta a presença desse achado em 9,8\% $(p<0,001)$, sendo, entretanto, similar aos dados de Cronemberg que revela presença da prega epicântica bilateral em 75,0\% da casuística $(p=0,236)$.

A presença de hipermetropia têm sido relatado em $100 \%$ da casuística, variando de $+0,50$ a $+6,00$ dioptrias, associada ou não ao astigmatismo ${ }^{(26)}$. Em outra série foi encontrado maior freqüência de astigmatismo (62,1\%), sendo por ordem decrescente o hipermetrópico composto (40,6\%), miópico composto $(16,2 \%)$ e o misto $(6,2 \%)^{(25)}$. No presente estudo, em concordância com os dados da literatura, foi encontrado maior freqüência de astigmatismo, presente em 58,9\% da casuística $(p=0,797)$.

Têm sido observadas associações da seqüência de Möebius com outras alterações sistêmicas e oculares menos freqüentes, tais como: catarata capsular posterior bilateral ${ }^{(28)}$, coloboma de íris ${ }^{(22)}$, hipogonadismo hipogonadotrófico com persistência de fibras de mielina peripapilares ${ }^{(27)}$, catarata congênita e coloboma retiniano ${ }^{(29)}$, nanismo pituitário e hipoplasia papilar associado à persistência de membrana pupilar e coloboma de nervo óptico ${ }^{(30)}$. Fundo de olho normal tem sido referido em todos os casos, com exceção de um caso com "fundus" miópico ${ }^{(25)}$. No presente estudo, essas observações não foram encontradas.

\section{CONCLUSÃO}

Foi observado, no presente estudo, que os pacientes portadores da seqüência de Möebius tiveram alterações oftalmológicas importantes. Sugere-se, portanto, que esses devem ser avaliados precocemente por um oftalmologista como forma 
de condução adequada, no que diz respeito à correção das ametropias, do desvio ocular, prevenção da ceratopatia de exposição e da deficiência visual.

\section{ABSTRACT}

Purpose: To evaluate the clinical ophthalmological findings in Möebius sequence patients. Methods: Twenty-eight Möebius sequence patients were studied at three ophthalmological centers in Pernambuco state, Brazil. Results: Bilateral epicanthus was found in 25 patients (89.3\%), ptosis in 14 (50.0\%), corneal opacities from exposure in 5 (17.8\%) and diminished Bell's reflex in 3 (10.7\%). Monocular visual acuity varied from 0.03 to 1 . Visual acuity deficiency was detected in 13 patients (46.4\%). The most frequent refractive error was astigmatism. It was observed in 33 eyes (58.9\%). At the primary position, 16 patients (57.2\%) had esotropia, 2 (7.1\%) had exotropia and 7 (25.0\%) had orthotropia. Fundoscopic examination was normal in 26 patients (92.8\%). Conclusions: It was observed that Möebius sequence patients presented important ophthalmological disorders, like visual acuity deficiency, incomitant strabismus, exposure keratitis and refractive errors. It is suggested that these patients should be evaluated early by an ophthalmologist for the diagnosis and treatment of these problems.

Keywords: Möebius syndrome/complications; Eye diseases; Eye/pathology; Visual acuity; Child

\section{REFERÊNCIAS}

1. Möebius PJ. Über angeborene doppolseitige Abducens-Facialis-Lähmung. Munch Med Wochschr. 1888;35:91-108.

2. Möbius PJ. Über infantile kernschwund. Munch Med Wochschr. 1892;39:17-58.

3. Miller MT, Sttrömland K. The Möebius sequence: a relook. J AAPOS. 1999;3(4):199-208.

4. Vasconcelos GC, Silva FBD, Almeida HC, Boas MLMV, Alvares MC. Síndrome de Möebius: achados clínicos e cirúrgicos em 7 pacientes. Arq Bras Oftalmol. 2001;64(3):211-5.

5. Ghabrial R, Versace P, Kourt G, Lipson A, Martin F. Möebius’ syndrome: features and etiology. J Pediatr Ophthalmol Strabismus.1998;35(6):304-11.

6. Baraitser M. Genetics of Möebius syndrome. J Med Genet. 1977;14(6):415-7.

7. Zitter FA, Wiser WC, Robinson A. Three-generation pedigree of a Möebius syndrome variant with chromosome translocation. Arch Neurol. 1977;34(7): 437-42.

8. Slee JJ, Smart RD, Viljoen DL. Deletion of chromosome 13 in Möebius syndrome. J Med Genet.1991;28(6):413-4.
9. Elsahy NI. Möebius syndrome associated with the mother taking thalidomide during gestation. Case report. Plast Reconstr Surg. 1973;51(1):93-5.

10. Kankirawatana P, Tennison MB, D’Cruz O, Greenwood RS. Möebius syndrome in infant exposed to cocaine in utero. Pediatr Neurol. 1993;9(1):71-2.

11. Courtens W, Vamos E, Hainaut M, Vergauwen P. Möebius syndrome in an infant exposed in utero to benzodiazepines. J Pediatr. 1992;121(5 Pt 1):833-4.

12. Gonzalez CH, Vargas FR, Perez AB, Kim CA, Brunoni D, Marques-Dias J et al. Limb deficiency with or without Möebius sequence in seven Brazilian children associated with misoprostol use in the first trimester of pregnancy. Am J Med Genet. 1993;47(1):59-64.

13. Pastuszak AL, Schüler L, Speck-Martins CE, Coelho KE, Cordello SM, Vargas F et al. Use of misoprostol during pregnancy and Möebius' syndrome in infants. N Engl J Med. 1998;338(26):1881-5.

14. Schüler L, Pastuszak A, Sanseverino TV, Orioli IM, Brunoni D, AshtonProlla P et al. Pregnancy outcome after exposure to misoprostol in Brazil: a prospective, controlled study. Reprod Toxicol. 1999;13(2):147-51.

15. Boudoux DD, Matos MAG, Gonçalves ED, Rocha M, Ventura LO, Hinrichsen SL. Síndrome de Möebius relacionada à ameaça de abortamento. Rev Bras Oftalmol. 2000;59(3):173-7.

16. Shepard TH. Möebius syndrome after misoprostol: a possible teratogenic mechanism. Lancet. 1995;346(8977):780.

17. Bavinck JN, Weaver DD. Subclavian artery supply disruption sequence: hypothesis of a vascular etiology for Poland, Klippel-Feil and Möebius anomalies. Am J Med Genet. 1986;23(4):903-18.

18. Marques-Dias MJ. Fisiopatogenia da síndrome de Möebius e da artrogripose decorrentes da exposição in utero ao misoprostol [tese]. São Paulo: Universidade de São Paulo;1999.

19. Ventura LMO. seqüência de Möebius: estudo comparativo das anomalias e distúrbios funcionais em crianças com ou sem uso de misoprostol durante a gestação [tese]. Minas Gerais: Universidade Federal de Minas Gerais; 2001.

20. Alves AA. Emetropias e ametropias. In: Alves AA, editors. Oftalmologia refração. Rio de Janeiro: Cultura Médica; 1994. p.69-77.

21. Soares EJC, Moura EM, Gonçalves JOR. Cirurgia plástica ocular. São Paulo: Roca; 1997.

22. Amaya LG, Walker J, Taylor D. Möebius syndrome: a study and report of 18 cases. Binoc Vis Quarterly. 1990;5:119-32.

23. Traboulsi EI, Maumenee IH. Extraocular muscle aplasia in Möebius syndrome. J Pediatr Ophthalmol Strabismus. 1986;23(3):120-2.

24. Henderson JL. The congenital facial diplegia syndrome: clinical features, pathology and aetiology. A review of sixty-one cases. Brain. 1939;62:381-403.

25. Cronemberger MF. Síndrome de Möebius: estudo das manifestações oculares, genético-clínicas, otorrinolaringológicas e cognitivas [Tese]. São Paulo: Universidade Federal de São Paulo, Escola Paulista de Medicina; 1997.

26. Vasconcelos GC, Silva FBD, Almeida HC, Boas MLV, Alvares MG. Síndrome de Möebius: achados clínicos e cirúrgicos em 7 pacientes. In: Congresso Del Consejo Latioamericano de Estrabismo;1998, Acapulco, México;1998. p.206.

27. Brackett LE, Demers LM, Mamourian AC, Ellenberger C Jr, Santen RJ. Möebius syndrome in association with hypogonadotropic hypogonadism. J Endocrinol Invest. 1991;14(7):599-607.

28. Torres RJA, Ferreira AA, Federmann I, Burnier Junior MNN. Dois casos de Síndrome de möebius: associação com catarata e achados histopatológicos. Arq Inst Penido Burnier. 1986;28(1):35-8.

29. Huerva V, Ascaso FJ, Palomar MT, Garcia J, Olivares JL, Gabas M, Palomar A. Syndrome de Möebius et colobome choriorétinien bilatéral. Ann Pediatr (Paris). 1992;39(5):313-6.

30. Hashimoto N, Sakakihara Y, Miki Y, Kagawa J, Egi S, Kamoshita S. Möebius syndrome associated with pituitary dwarfism and hypoplastic optic disc. Acta Paediatr Jpn. 1993;35(2):144-7. Review.

\section{Ao enviar um artigo para publicação,}

leiaATENTAMENTE as instruçóes para autores,

constante no final de cada fascículo. 